\title{
VIII. EMOTION AND THE ROLE OF THE BODY IN MENTAL LIFE
}

\author{
REGINA PALLY
}

\begin{abstract}
"[By] means of my psychical work, I have had to overcome a psychical force in the patients which was opposed to the pathogenic ideas becoming conscious [...] They were all of a distressing nature calculated to arouse the affects of shame, of self-reproach and of psychical pain and the feeling of being harmed; they were all of a kind that one would prefer not to have experienced, $[\ldots]$ that one would rather forget."
\end{abstract}

(Freud 1895d, pp. 268-269)

\section{INTRODUCTION}

Since its inception, psychoanalysis recognized the close connection between mind and body, psyche and soma (Freud 1950a [1895]). Although Freud believed in a biologic basis for psychological phenomena (Freud 1950a [1895]) he was unable to demonstrate it, given the limited scientific knowledge of his time. Pyschoanalysis therefore welcomed the decade of the brain, with its rich discoveries, many, of which support and enhance psychoanalytic ideas. Using current neuroscience data, I intend to show that the mind and body are linked through the activation of emotion.

\section{THE NEUROSCIENCE MODEL OF EMOTION}

Embedded within emotion itself is the organization of adaptive behavior (Damasio 1995; LeDoux 1994, 1996). More specifically, emotion organizes the behaviors the animal will need to adapt to its environment and organizes all the necessary brain and body changes to enact that behavior. In emotion, mind and body ${ }^{1}$ join forces to generate and sup-

\footnotetext{
${ }^{1}$ The body includes the brain as well as the body proper.
} 
port adaptive behaviors. Body, refers to the body proper with all the internal viscera, including the brain.

Neuroscience agrees with the psychoanalytic view that a behavioral response, which may have been adaptive in one situation, may prove maladaptive in another. For example, one woman patient, a business executive, who as a child had an over controlling parent, learned to cope by being overly compliant to avoid conflict. However, later on, in the workplace, her over-compliance inhibits her success in a competitive career. Another area of compatibility is that both neuroscience and psychoanalysis recognize that the link between an emotion and its ensuing behavior is often outside of conscious awareness. A person can be afraid and run, or can be ashamed and lower their head and fall silent, without even being at all conscious of their feeling. By becoming more consciously aware of the link, the person has a greater ability to shift to a more adaptive behavior. For example one patient, a tenured professor at a major university, who was not aware of his deep need for admiration, casually hands me his curriculum vitae, and says "I thought this might be of interest to you" and quickly proceeds to talk about some problem with his wife. When I comment on the CV, he falls silent. As we explore what happened, he tells me how mortified he now feels, since he had been completely unaware that the $\mathrm{CV}$ was his way of seeking out my admiration for his accomplishments. He had repressed this longing as a result of how foolish his father made him feel for showing off.

Embedded in the neuroscience model of emotion are three interrelated assumptions, that I will emphasize throughout the paper. 1. For optimal health emotion must be regulated, 2. Most emotion occurs without conscious awareness 3 . Being conscious of emotion helps regulate emotion.

For optimal health emotion must be regulated. Emotion follows a natural progression from reaction to recovery. Emotion is regulated because after an emotion is activated, brain and body feedback systems automatically activate the mechanisms by which we recover from that emotion and return to baseline (Gunnar 1992; Degangi et al 1991).

Feedback systems are designed so that emotion occurs within an optimal range - not too much and not too little. Emotion must be regulated so that we can cope with the environment yet maintain sufficient homeostasis to support health (Davidson, Jackson et al. 2000; Davidson, Marshall et al. 2000; Silk 1998, 2000). To react emotionally is not only automatic, and involuntary it is necessary and adaptive adaptive. Pathology occurs when patients are not able, to return to a baseline state of 
calm following emotional arousal. One woman patient, the business executive, when she suffers a disappoint in a relationship or career goal becomes so mired in disappointment, she loses all enjoyment in her usual activites. Another male patient, a stock broker, reacts with rage to a personal slight by his wife. He withdraws and sulks, to punish her for days.

Most emotion occurs without conscious awareness. We only become conscious of a small percentage of emotion, which neuroscientists refer to as 'feelings'- sadness, joy, fear and the like (Damasio 1994, 1999). Some individuals only consciously process the body aspect of emotion. (Taylor 1992) These individuals may exhibit alexithymia and be susceptible to psychosomatic and somatizing disorders.

Being conscious of emotion helps regulate emotion. Although most of the time emotion is non-conscious, consciousness of feelings can facilitate voluntary control over how the emotion is expressed - for example, in the case of the stock broker, not acting on a behavior associated with a feeling, such as not sulking he feels slighted.

\section{AN EVOLUTIONARY PERSPECTIVE}

It is not known exactly when emotion first evolved, but we do know that it begins to flourish with the emergence of mammals and the expansion of the limbic system (LeDoux 1996). Humans share much of the same emotional circuitry with other mammals, which is why neuroscientists believe animal emotion is a good research model for human emotion.

Without emotion, lower animals are limited to a few reflexive responses to stimuli. Emotion enables mammals to respond more flexiblly to the same stimulus in a number of ways, depending on the whole context. This reaches its highest degree in humans. Touch the lowly Aplysia snail, for example, and all it can do is reflexively withdraw its gill and mantle, no matter WHO touches it, or HOW. Touch a human, and depending on which emotion is genereated, the person can run away, fight, hug, laugh, or make love...depending on WHO the touch is from (a stranger, a friend, a lover) and HOW the touch is delivered (rough, gentle, ticklish). The large number of possibilities is what enables humans to have such complex and varied social and cultural relationships.

The most primitive emotions are essentially positive and negative hedonic tone, and the behaviors that ensue are essentially ap- 
proach/avoid reactions. In other words - if it is good, approach it; if bad, avoid it. This is essentially what the psychoanalytic concept of pleasure/unpleasure is derived from at the biological level. Babies are born with this very primitive emotional plan. A number of other emotions emerge early in the first year of life, such as fear, sadness, surprise and joy. The 'social' emotions, such as guilt, embarrassment, and pride develop by about $2-3$ years. As language develops emotions become more complex. Examples are distinctions such as pride in ones country, verses pride in ones children, or sadness over losing a friend, verses sadness over losing a parent.

Emotion can be either inborn or learned. According to Ekman and Panksepp (Ekman 1993; Panksepp 1998) there are several innate, inborn 'basic' emotions. For Ekman they are anger, fear, sadness, disgust, surprise and joy. For Panksepp they are rage, fear, panic, lust, seeking, play and care. Each emotion is presumed to have its own brain circuitry and physiology. Complex emotions are learned from experience and probably do not have a specific circuitry.

\section{THE FUNCTION OF EMOTION}

Emotion functions to organize the mind and body toward a common goal or purpose, by coordinating the mind and body to support the behaviors which best achieve the animal's goal (LeDoux 1996; Panksepp 1998). The goal may be to find food, or to mate, or explore or find a shady spot to relax in. With an emotion all parts of the mind and body are playing on the same 'team', meaning that perception, memory, thoughts, decisions, behavior and body physiology are coordinated to help the animal meet its goal. In a state of FEAR, the MIND and BODY coordinate. The mind is vigilant, on alert for danger, more likely to perceive danger, have frightening thoughts, and remember scary events. The body is able to run away because there is an increase in heart rate, blood pressure, respiratory rate, tone of the muscles and blood flow to the muscles.

In the clinical setting, emotion organizes the transference relationship. A particular emotion coordinates the patients perceptions, memories, associations, behaviors and visceral response. For example, the emotion of anger over not being taken seriously is triggered in the transference. The patient's glaring facial expression, tense body posture and harsh tone of voice signal anger. Their memories and associations will 
include incidents with this same emotion. They will perceive the situation between the two of you as one in which you do not take them seriously. Shame in the transference will organize a different set of responses. Love organizes yet another set of mind and body responses.

\section{EMOTION MUST BE REGULATED}

Specific neurobiologic systems are in place, both for the reaction phase of emotion and for the recovery phase. Once an emotion is generated and expressed, it naturally dissipates on its own. It is often very helpful, simply to remind patients that feelings tend to fade on their own. This reduces anxiety and distress for those who believe their bad feeling will last forever.

Dysregulated states of emotion can occur. Either not enough emotion is generated, or too much is generated or recovery fails to occur. Many of the clinical situations we treat are the result of dysregulated emotion. Dysregulated emotion effects the body as well as the mind. Dysregulation of emotion can be due to inborn traits, insecure attachment, physical or sexual trauma, and neglect or prolonged separation (Silk 2000). Infants have some inborn capacity for the self-regulation of emotion, but are highly dependent on caretakers in this way (Beebe \& Lachmann 1988; Stern 1985; Beebe \& Lachmann 1998). Eventually, as they mature, they develop more and more capacity for regulating their own emotion. However, all humans remain dependent, to some degree, on others for affect regulation.

\section{NEUROANATOMY AND NEUROBIOLOGY OF EMOTION}

The brain regions involved with emotion are the limbic system, hypothalamus, [HYPO] and the brain stem, [BS] (LeDoux 1996; Damasio 1999; Schore 1994; Panksepp 1998). The limbic system includes the amygdala, $[\mathrm{AMG}]$, anterior cigulate, $[\mathrm{AC}]$ ), and a "limibic extension" into the cortex, the orbitofrontal cortex $[\mathrm{OFC}]$. These brain centers stimulate the production of neurotransmitters and hormones, which run the circuitry of the brain and the body's physiologic processes.

Stimulus inputs come into the brain's sensory cortex and are funneled to the AMG, and the OFC..$^{2}$ The AMG and OFC appraise those

\footnotetext{
${ }^{2}$ Appraisals are for the most part non-conscious.
} 
stimuli as to their relevance to the individual. Once relevance is determined, an emotional response is generated by the AMG and OFC via signals to the HYPO and BS. The response output consists body physiologic changes and behaviors. Stimulus inputs come into the brain from external sources (i.e. the world), through the five senses (i.e. sight, sound, taste, touch and smell) as well as from internal sources, (i.e. 'somato-sensory' sources, including heart rate, respiratory rate, gastric motility, and blood pressure). Outputs go back, outwardly to the world and, inwardly to the body in the form of motor behavior (such as waving, smiling, talking, running), and changes of body physiology (such as blushing, sweating, crying, sighing, muscle tension). What these details highlight is the cyclic nature of the system. Response outputs themselves, can become new stimulus inputs. For example we sense our own flush of embarrassment and feel more embarrassed.

Emotion occurs every time we perceive a situation, whether or not that situation is the simple sound of a bell, or the sound one hears while listening to a Beethoven sonata. Let me emphasize - ALL experience activates emotion which is generated automatically and involuntarily, and the vast majority of the time with out conscious awareness of the emotion. Again let me emphasize - We have very little voluntary control over what emotion is generated and what body changes occur. We do however have some voluntary control over, certain aspects of the behaviors which arise. In treatment this is valuable to explain to patients. Many patients feel either guilty or ashamed of the feelings they have, as if somehow they are accountable or responsible for how they feel. Neuroscience suggests otherwise. All we can be held responsible for is HOW we behave in the presence of those feelings. In many respects this is what Freud set out to do with sexual desire and aggression. Sexual and aggressive desires are unavoidable, normal human drives. If there is excessive guilt or anxiety, they may be too heavily defended against and will be expressed in maladaptive behaviors. Psychoanalysis aims to modify guilt and anxiety which leads to more adaptive sublimated behaviors.

\section{1. THE FIRST PHASE OF EMOTION: APPRAISAL OF STIMULI}

Each of the five senses consists of a specialized sensory end organ (i.e., retina, cochlea) which detects sensations from the external world and sends this information, via the thalamus, to the AMG, sensory cortex and OFC. In the 'somato-sensory system, specialized sensors in the 
internal organs send signals about the state of the body ${ }^{3}$, via the thalamus, to the AMG, sensory cortex and PFC. Appraisals are made about these sensations with respect to their relevance to the organism. Very simple appraisals are made at the level of the $\mathrm{AMG}^{4}$. These can be thought of as rough assessments of the situation. Is the situation 'good vs bad?, pleasurable vs unpleasurable?, safe vs dangerous?, rewarding vs frustrating. More complex appraisals are made at the level of the $\mathrm{OFC}^{5}$, which can be more subtle, not so black and white. An example of a complex appraisal would be 'The cake tastes good, but I need to lose weight so it is bad for me now.' Or 'It's a beautiful day for a walk in the woods, but I probably should put that off until after I finish studying.' It must be emphasized however, even though I have written these appraisals out, as if the person is thinking out loud, appraisals are generally not conscious. A child with a depressed mother can learn what facial cues on mother's face signal 'stay away now! I won't respond to you emotionally' vs 'I am OK and available to you emotionally', without ever being consciously aware of such an assessment. Similarly a patient who reads into the analyst's body language a disapproval can become quiet, without awareness of the reason for the silence. Neuroscience therefore alters somewhat the psychoanalytic view of resistence. Sometimes patients do not know why they behave or react a certain way not because of resistence, but because there are many brain processes, such as appraisal, which often are just not conscious.

Appraisals can be innate or learned. Innate appraisals are built into the brain as a result of genetic programming. A newborn baby innately gives more value to faces than non-faces. A young child is afraid when a balloon pops, even if they have never been exposed to any dangerous loud noises before. But most appraisals are learned. We have to learn that running into the street is dangerous or that touching a hot stove is dangerous. Generally speaking the AMG can handle simple learned appraisal. When, however, there are a number of possible appraisals to choose from, it is the OFC that is required to select the most adaptive for the current circumstances. A situation in which the mother's mouth is

${ }^{3}$ The state of the body involves physiologic sensations, vibration, body position, balance, muscle tension, and chemical levels (i.e., oxygen, $\mathrm{CO} 2, \mathrm{pH}$ and glucose)

${ }^{4}$ AMG: a subcortical structure, deep within the medial temporal lobe, near the hippocampus.

${ }^{5}$ OFC: essentially a 'limbic' extension into the frontal lobe and part of the prefrontal cortex, which is the seat of higher cognition and executive control functions. 
smiling, but her tone of voice, indicates she is angry, and her eyes indicate that she is sad, requires the kind of selective choice abilities of the OFC. Even a young child can learn not to run into the street, because the AMG is fully developed by that age. But to make sense of which facial signal is the relevant one to respond to can be quite confusing for a young child, because the OFC is immature. In fact the OFC along with the whole PFC does not fully mature until well into the 20 's. The late maturation of the PFC explains why children are poor at reasoning, judgement, planning and affect regulation.

\section{2. THE RESPONSE OUTPUT PART OF EMOTION}

Once an appraisal occurs, the AMG and OFC signal the HYPO and BS to activate an emotional response, consisting of three objectively measurable 'biologic' parts, and a 'subjective part'. The biologic responses involve the body's biochemistry, autonomic nervous system and motor behavior, which can all occur without conscious awareness. The subjective part is conscious and is called feelings. It is presumed that the biologic changes contribute to the subjective feelings, but the exact mechanisms have not been fully worked out yet.

\section{2. 1. Biochemistry}

Neuroepinephrine $[\mathrm{NE}]$ is the first initial biochemical response activated by the emotional system (LeDoux 1994, 1995). The NE response is an 'arousal' response, a rather non-specific reaction to any kind of emotionally significant stimulus. NE causes an 'alerting and orienting' to the source of the stimulation, enhances attentional focus to the relevant stimulus and makes the brain more ready to receive incoming stimuli. For example a herd of animals all perk up their ears and turn them in the direction of a sudden sound. A crowded auditorium will all turn to the direction of a sudden noise. NE also increases activity of the sympathetic nervous system (SNS) to dilate the pupils, increase heart rate and blood flow to the muscles. Finally NE activates the AMG to signal the hippocampus [ HIPPO] 'This is significant. Remember it!'

Cortisol, the stress hormone, is released after NE, if the situation is stressful. The HYPO activates the production of cortisol, via a long and complex sequence of events, referred to as the hypothalamic-pituitaryadrenal axis [HPA axis] (Panksepp 1998). The HYPO produces cortisolreleasing-factor $[\mathrm{CRF}]$ which signals the pituitary to produce adreno- 
cortico-tropic hormone $[\mathrm{ACTH}]$, which signals the adrenal gland to produce cortisol, which then disperses through the blood stream and can affect all parts of the body. Production of cortisol is very well regulated. In a delicate negative feedback loop, cortisol is detected by cortisol receptors in the brain, most importantly in the HYPO, HIPPO and AMG. This negative feedback system signals the HPA axis to shut off the production of cortisol.

Cortisol helps an animal cope with stress. Cortisol provides the kinds of physiologic changes in the body necessary to support coping behaviors, such as running or, fighting. Specifically cortisol increases blood pressure and increases blood glucose to make more glucose available to muscles for energy. Cortisol is also part of a feedback system for NE. Cortisol acts to shut off the release of NE.

The HPA axis and the cortisol respone must be in the optimal range. Hyper-reactivity of the HPA axis, can occur as a result of early childhood truama, prolonged separations and neglect (Teicher 2000; Yehuda 2001). Very high levels of cortisol are harmful. Very high levels of cortisol, can atrophy or even destroy cells in the HIPPO (Bremner, Randal et al. 1995; Sapolsky 1996). Such damage, can lead to impairments of memory. Since cortisol receptors in the HIPPO help shut off cortisol, damage to the HIPPO can contribute to excessively high cortisol levels. The damaging effect of cortisol on the HIPPO helps explain why with trauma there may be reduced rather than increased memory for the traumatic situation as one would expect. Indeed what we often find in psychoanalytic patients who have been traumatized, by physical or sexual violence, their memory is spotty for childhood events. This may seem like repression, or dissociation, but may in fact be the result of damage to the memory system itself. Also because damage to the HIPPO can impair cortisol regulation, these patients often react, even to benign situations, as if they are overwhelming and stressful. Chronic hyper-reactivity of the HPA axis, predisposes these individuals to depression and anxiety, presumably due to chronic high levels of CRF (Silk 2000; Yehuda 2001).

On the opposite end, too little cortisol can be harmful. For reasons not yet understood, the neurobiologic profile of individuals who suffer from post traumatic stress disorder, PTSD, appears to be - hyperreactivity of the HPA axis, with high levels of CRF but a blunted cortisol response, - i.e. the cortisol rise during traumatic or stressful events is less than expected. This profile, may be the factor that predisposes individuals to develop PTSD and can explain the often contradictory 
symptoms of PTSD. With a blunted cortisol response, NE arousal does not shut off. PTSD patients show hyper-arousal, vigilance, increased startle (e.g,, alerting and orienting response), and heightened memory for the traumatic event in the form of flashbacks ${ }^{6}$ (Van der Kolk 1993). The part of PTSD characterized by emotional detachment, may be due to the low cortisol itself. Low levels of cortisol have been found to be associated with impaired transfer of information from the right hemisphere (associated more with emotional processing) to the left hemisphere (associated more with verbal processing). Consequently these patients appear emotionally detached, because they can't verbalize their feelings (Henry 1993). An unfortunate byproduct of this reduced expressiveness is that these individuals may be unable to adequately use other individuals to help them reduce their distress. One woman patient, a graduate student, suffered neglect, because her mother was chronically depressed. She suffered trauma, from her parent's talk about their holocaust experiences and because she was sexually molested at 8 years old, by a male cousin. She suffered flashbacks, felt chronically stressed, and overwhelmed, but she was completely detached from any awareness of emotional distress about what had happened to her as a child. She kept her distress to herself most of the time. In the transference, she was very warm and accepting of me. If I did anything wrong, or made any mistakes she attributed it to how many responsibilities I had manage and was forgiving because I helped so many people. She had no ability however to forgive herself, or give herself any warmth or empathy for what she had been through and had to cope with as a child, because she could not get in touch with the idea that anything terrible had happened to her. This kind of emotional detachment is problematic in the psychoanalytic treatment situation. She could not feel any awareness of transference feelings. As is well known, unless a person can feel the emotion within the transference, (i.e., fear, or dependency), interpretations will be ineffective.

Endorphins: Endorphins, the brain's endogenous opiates, are hormones, released by the HYPO, as well as other brain regions, which reduce pain (both physical and emotional), create a sense of well being,

\footnotetext{
${ }^{6}$ Memory for events normally goes through, at least, some degree of fading. In the case of PTSD, instead what occurs is memory does not fade, and the person has flashbacks- the intense, vivid recall of traumatic events that are triggered very easily and do not appear to fade.
} 
and promote mother-infant attachment (Kalin, Shelton et al. 1995; Panksepp 1998). Because endorphins are released during pain, individuals can become 'addicted' to practices such as cutting themselves.

Mother-infant attachment involves two emotional processes, separation distress and reunion comfort. Each of these emotions involves different brain chemistry (Hofer 1996; Meaney 2001; Panksepp 1998; Suomi 1999). Separation distress is mediated by the HPA axis. During a separation, CRF is released, leading to increased cortisol. When the baby is reunited with the mother, reunion comfort is mediated by endorphins. Reunion with the mother increases endorphin levels in both the baby and the mother! By shutting off cortisol, endorphins shut off the separation distress response. The endorphins give both baby and mother a feeling of well being and decreased emotional pain. Babies, in a sense, become addicted to their mothers. This may help explain the insatiable need of certain patients to find a mother figure in the analyst. But mothers also gets 'addicted' to their babies as well, which may underlie what Winnicott calls Primary Maternal Preoccupation \{ref\}.

Experiements on monkeys (Kalin, Shelton et al. 1995) where endorphin levels can be easily manipulated, illustrate a number of clinically relevant points. When endorphins are pharmacologically blocked, in the mother and infant, both engage in more intense reunion behavior, (which consists of a kind of hugging and vocalizing), ostensibly to increase their endorphins. If on the other hand, exogenous endorphins are given in the form of morphine, both decrease their reunion behaviors, to down regulate endorphin. This data suggests why individuals with early separations and loss may fail to have adequate endogenous endorphins and may be more susceptible to opiate addiction, and more susceptible to excessive dependency in the transference situation. This data also explains why heroin or morphine addicts make such poor parents. They do not react properly to the infant's need for contact.

Oxytocin is a hormone, released by the pituitary gland, during reproductive behaviors such as, sexual activity, labor and nursing (Insel 1997). The oxytocin released during sexual acitivity enhances monogamous pair bonding between mating partners and parental care toward offspring. If the action of Oxytocin is blocked, both monogamy and parental care are impaired. What this suggests is that sexuality in humans serves not just reproductive functions, but enhances the feelings of attachment between the partners and also enhances their desire to care for their children. We observe this clinically when couples are not having sex, they are very often emotionally distant from each other. As 
analysts we tend to think of the problem as one in which their emotional conflicts are getting in the way of a good sexual relationship. And while this is definitely an important factor to consider, it also appears that the sexual connection itself, serves an attachment function, and may be what helps partners feel close and committed to one another.

\section{2. 2. Autonomic nervous system}

The Autonomic Nervous System [ANS] carrys sensory and motor information between the brain and the internal viscera (Schore 1994). The HYPO is the brain center which regulates the ANS. The ANS is divided into the Sympathetic [SNS] and Parasympathetic [PNS] nervous system. ALL organs of the body are innervated by branch from the SNS and PNS. The SNS and PNS each have an opposite effect. The SNS regulates body organs to cope with external stress, and the PNS regulates body organs to relax, regenerate and repair. The SNS is often referred to as the Fight or Flight system and the PNS as the Rest and Digest system.

Much of the emotional response is the result of ANS activity. For example with fear or anxiety the heart races, the blood vessels pump, the mouth turns dry, the stomach slows down (which may cause nausea or decreased appetite), and the large intestine may speed up (which may cause cramps and diarrhea). Much of what we show in our facial expression of emotion is the result of ANS activity. The face flushes with embarrassment, or shows pallor with emotional 'shock'. The pupils dilate with fear. The eyes tear with sadness. Even our voice shows emotion as a result of ANS activity. The vocal cords quiver with fear and anxiety. Disgust, as an emotion, most likely began as a way of getting rid of spoiled food. When disgust is directed, not at a food, but at a person's behavior, our ANS may activate in the same way, with a slowing of the stomach and even some reverse peristalsis.

Somatizing and Alexithymia Neuroscientists reserve the word 'feelings' for the conscious subjective experience of emotion. Some individuals only experience the 'somatic' aspect of emotion. These individuals fail to feel emotions as subjective feelings, and have difficulty putting their emotions into words. A person who 'somatizes' may complain of headache, or dizziness, or stomach pain, or 'a lump in the throat' or chest pain, but will not connect these somatic complaints with feelings of fear or anxiety. It appears clinically that such patients are often poor analytic or even psychotherapy candidates because they have 
difficulty reflecting on their subjective emotional state and talking about feelings. This condition has been called Alexithymia (Krystal 1974). Education is helpful to enable these patients to engage in psychotherapy. Education consists in explaining that all emotion has a somatic part; and that what they are experiencing in their body is the result of emotion (Taylor 1992). They need to re-interpret their physical symptoms as an emotional response and to put words to those emotions. It appears that the process of putting words to emotions helps shift the experience away from the body and into subjective feeling states where they can be talked about. Clinically we all observe that simply talking about upsetting feelings can be calming and helpful, even if no 'analytic interpretation' occurs. It may be that talking, engages conscious self reflection, represents experience at the symbolic abstract level, and activates the highest levels of the brain, the PFC. The orbito-frontal region of the PFC (the $\mathrm{OFC}$ ) is the highest center of affect regulation and is crucial in being able to experience situations from alternate perspectives.

Anxiety Disorders: The emotions of anxiety and fear have a similar biologic profile (Davis 1992). When the AMG appraises a stimulus as dangerous or associated with danger, (in the way that the sound of a snapping twig can be associated with the approach of a tiger), the AMG can trigger fear or anxiety. The AMG signals the HPYO and BS to produce a whole host of body responses. NE is released producing vigilance. SNS activity produces sweaty palms, difficulty swallowing, heart palpitations, light headedness or dizziness, difficulty breathing, queasiness and erectile difficulty in males. Increased tension in skeletal muscles to enhance running or fighting, can lead to complaints of chest pain, from tightening of the intercostals muscles, and headache, from tension in scalp muscles. In fact what many 'somatizers' report as physical symptoms, are essentially, the physical accompaniments of anxiety and fear.

Panic attacks are a severe form of anxiety. In panic, the patient has a sense of dread or impending doom and difficulty breathing. Neurimaging studies using fMRI show increased activity of the AMG. ${ }^{7}$ Since even

${ }^{7}$ FMRI is a neuroimaging device which measures blood flow to various brain regions. Blood flow brings in oxygenated hemoglobin and serves as indirect measure of brain metabolism. Increase blood flow to a region, indicates increased metabolic acitivity of that region. Neuroscientists use this kind of data to localize what brain region is involved with a particular mental process. For example on memory tasks the fMRI shows increased activity in the HIPPO. 
the physiologic changes associated with anxiety, such as increased heart rate, can be unconsciously appraised by the AMG as 'danger signs', even low levels of anxiety can escalate into panic states.

The treatment of anxiety follows from the biology. Pharmacologic treatments involve benzodiazapines [BNZ] and selective serotonin reuptake inhibitors [SSRI's] both act either directly or indirectly to inhibit the reactions of the AMG. Cognitive Behavior Therapy [CBT] focuses on the appraisal aspect of emotion - using voluntary conscious appraisals to counteract automatic non-consciously derived ones. This engages the PFC which has more ability to select a more adaptive response and inhibit an automatic maladaptive one (Panksepp 1998). For example, an anxious person can become more anxious because their appraisal system signals 'Danger! Heart is racing'. Patients can be instructed to notice heart palpitations and consciously assign them a more benign appraisal value. "When my heart rate goes up it just means I am anxious. It does not mean I am having a heart attack!" Although many analysts do not recognize it, CBT-like techniques are used in transference interpretations. I would often say to my patient the professor, who felt his mother was cold and did not love or care about him. She could emotionally 'ice' him out. Once she locked him out of his house by his mother, when he was 10 years old, because of a prank in which he scared her with a plastic spider. "When I don't talk, it is as if you assume I don't care about you; I have locked you out. But it could mean I am intently listening and do not want to interrupt what you are saying" A more benign appraisal can keep anxiety at more manageable levels. Since severe anxiety interferes with memory and cognitive functioning, reducing excessive levels of anxiety, in the case of analysis, promotes greater tolerance for the treatment and more ability to reflect on the process.

Chronic Stress. Stress is related to situations of danger and threat, and can either be acute (for a short time) or chronic (for a long time). An acute stress response is healthy and adaptive because it enables the individual to cope with the stress producing situation. Chronic stress however can be harmful both physically and emotionally (Sapolsky 1996). With chronic stress, the initial acute phase does not return to baseline 'homeostatic' levels. During during chronic stress, there are chronically high levels of SNS activity, CRF and cortisol, which can potentially cause physical and emotional illnesses, or exacerbate existing ones including, hypertension, heart disease, an altered inflammatory or immune response, asthma, bone resorption and osteoporosis, Diabetes Mellitus, Depression Anxiety (Nemeroff 2002). Chronic stress in early 
life, i.e. childhood neglect and abuse, (physical and sexual) can lead to life long, permanent hyperactivity of the HPA axis (Meaney 2001; Newport, Stowe et al. 2002). When these children grow up they are more likely to develop depression, and anxiety as well as many of the physical conditions mentioned above.

\section{2. 3. Motor behavior}

Motor behaviors which result from emotion are generally activated involuntarily and without conscious awareness. The most common emotional behavior is the 'freeze response', the sudden stillness that occurs as an animal first detects danger (i.e., the 'deer in the headlights' reaction). Others include running, hitting, hugging, waving, facial expression and changes in posture. A tall erect stance is associated with pride, while a 'slumped down' posture is associated with sadness. The facial expressions of emotion involve movement of the muscles of the face. Different muscles groups of the face are contracted to produce frowning (anger), smiling (happiness) raising the eye brows (surprise), curling the lip (disgust). Contraction of the eye muscles alters direction of gaze. Eyelids and eyes are lowered in shame, staring straight ahead is associated with anger, gazing into the eyes of a loved one is associated with love.

Each of Ekman's (1983, 1990, 1993) six 'basic emotions'- anger, fear, sadness, disgust, happiness and surprise - has a characteristic facial expression, specific muscle groups that contract in the face and a characteristic profile of ANS activity that corresponds with each emotion. The biochemistry, ANS activity, motor behavior (i.e., facial expression) and subjective feelings of emotion are linked. In experiments, subjects either see emotion laden images, or imagine an emotion or are taught to contract the muscles groups of their face which correspond to an emotion. ANS measures are taken, such as heart rate or skin temperature. When subjects either watch images or imagine a emotion, their ANS shows the characteristic profile. But also, many subjects who simply contract the appropriate facial muscles, even though they do not know what emotion this corresponds to, also have the ANS and subjectively feel the feeling!

When two people converse, they automatically and unconsciously match each other's non-verbal behavior, including facial expression, gesture and body posture. Studies show that the degree to which people match correlates with their sense of rapport and liking each other- even though the process of matching is entirely outside of conscious aware- 
ness. In an experiment by Feldstein \& Welkowitz (1978) two complete strangers are asked to talk with one another and afterwards each is asked to rate whether or not they would be interested in going out for dinner with the other person. Their conversation is recorded on videotape. When there is a high degree of matching within the dyad, they are both more likely to want to meet up with each other again, than in dyads where matching is low. Studies between hospitalized patients and therapists indicate that when the patient and therapist are high in matching, the therapist is more likely to have a positive feeling toward the patient. It is theorized (Beebe \& Lachmann 1988) that matching contributes to empathy. Since a person's facial expression is linked to a characteristic pattern of ANS activity, when we match the facial expression of another, we set up within ourselves the same body state that they have... and we literally can feel what they feel.

Within a dyad there can be complimentarity, in which each member displays a complimentary behavior. In matching, behavior is similar. When the baby coos, the mother coos. In complimentarity, if the baby cries, the mother picks up the baby and soothes it, i.e., she does not cry herself. There are a number of implications of matching and complimentarity for, psychoanalysis (Beebe \& Lachmann 1988). Such processes occur without conscious voluntary control over the behavior. One matches or reciprocates automatically and without any thinking or planning involved. Matching may explain why the analyst often feels they know what is going on in the patient, simply from seeing them in the waiting room. Once the patient is lying on the couch, the main source for non-verbal matching, i.e., the face, is lost. Except for vocal quailities such as tone, timbre, rhythm and pitch, other sources are diminished, such as body movement and posture, although more so for the patient than analyst. The decreased ability for non-verbal matching, albeit that it occurs outside conscious awareness, may explain why some patients on the couch have such a hard time feeling the analyst is 'there' in a caring way.

The treatment of a professional woman in analysis, illustrates matching and complimentarity (Pally 1996, 2001). During her childhood, she suffered many long absences from her depressed mother, who was hospitalized on several occasions. The woman had two different ways of talking. She spoke like a competent, independent minded and self sufficient professional eager to work analysis. At other times she seemed small and fragile, anxious about beginning analysis, for fear of the unknown, of getting hurt, or of discovering something frightening. 
In the first several months of the analysis, I began to notice myself talking in a rhythmic, soft, high pitched voice as if I was a mother with a baby. In the transference she began to feel completely protected by me, as if in a "safe bubble". In the countertransference, I felt like a mother in a state of "primary maternal preoccupation" (Winnicott 1956). I was matching her the little baby vocal rhythms and pitch, with my mothering vocal rhythms and pitch, and I was providing the complimentary response to her distress with rocking motion to comfort and soothe her.

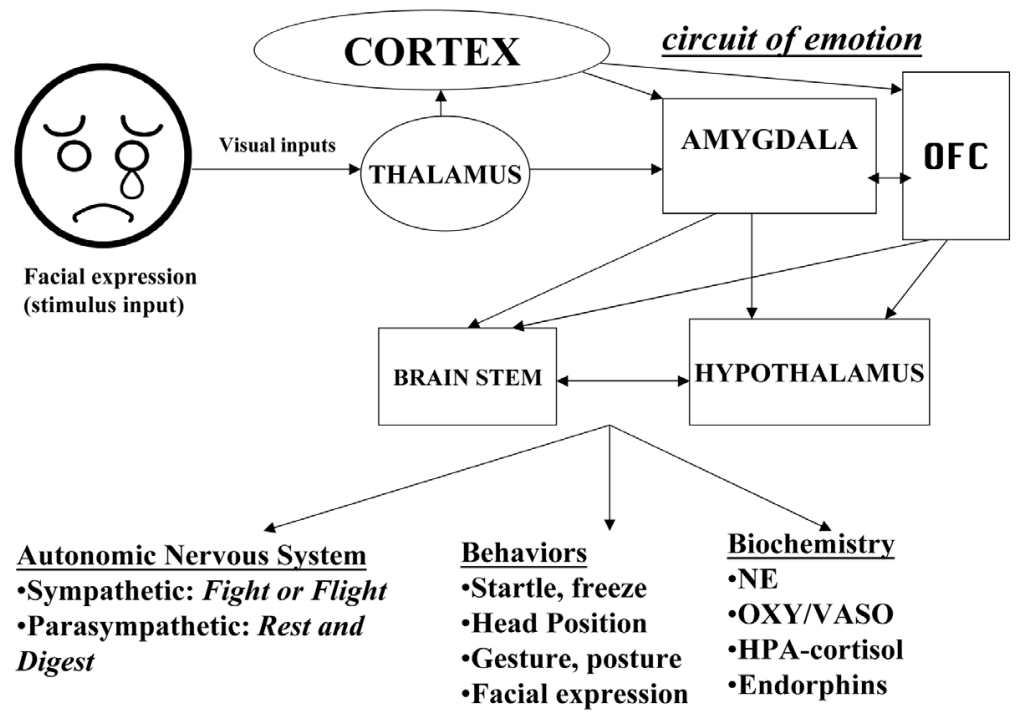

\section{CLINICAL IMPLICATIONS}

\section{1. EMOTION KEEPS US ORGANIZED}

We react emotionally to the environment, because emotion organizes the brain and body to perform the behaviors necessary to cope with environmental situations. We must react emotionally, for physical survival, and, because we are such highly social creatures, for surviving within our social network. For example, shame is generally induced in a person, when their exploratory behavior or exuberance is abruptly interrupted (Schore 1994). Shame can occur when a child is chastised by its mother for climbing up on a shelf to see what is there, with "STOP! You 
will FALL!" or for running into a room full of relatives to tell mother about what happened at school and being suddenly told to "Stop! Please greet Auntie Jane!" The emotion of shame 'organizes' the necessary adaptive response to these abrupt prohibitions. Shame is designed to inhibit exploratory behavior and dampen enthusiasm. Shame involves increased activity of the PNS (ie, which slows the heart rate, decreases blood flow to the muscles) to support the process of withdrawal and behavioral inhibition. Behaviorally, the child stops their behavior and dampens outward signs of enthusiasm, i.e., lowers the head and eyes, and may even withdraw or hide. Interestingly, shame does not develop, until the second year of life, perhaps because this when when the child develops sufficient locomotor abilities and the motivation to exuberantly explore its environment. One way to look at the adaptive value of shame, therefore, is that shame helps us to learn not only rules of safety, but also, the rules of society. Each social group and culture has its own norms. Adaptive functioning requires us to learn these rules. Those that don't are marginalized. We learn these rules which govern our social behavior, as a type of procedural memory; meaning that once it is learned it can be activated unconsciously and automatically (Grigsby 1994).

\section{2. EMOTION MUST BE REGULATED}

Emotional regulation means two things. One, we REACT emotionally and RECOVER afterward, i.e., return to baseline. Two, emotion occurs within an 'optimal range of intensity'. Individuals who are not well regulated often suffer physical and psychological conditions, such as somatic symptoms, anxiety, depression and 'character' traits.

Shame, guilt, fear and anxiety are all good examples of how too little emotion or too much emotion is not good. Sufficient shame is necessary for learning the social norms of how to relate to others. Therefore, civilization' depends, on shame as much as it does on guilt. In the clinical situation we often see patients who don't 'read' or respond properly to social boundaries. Some are too intrusive, as occurs with borderline patients. Some are too demanding of attention, as occurs with narcissistic conditions. These individuals have difficulty with friends, family and colleagues. In the transference, the intrusive ones make us feel invaded, the attention seeking ones, make us feel used. Perhaps these individuals were raised by parents who did not provide appropriate 'shame guidance', to learn the proper social rules regarding distance and attention. 
Perhaps these individuals grew up with parents who did not know these rules and thus passed these traits on to their children, as a form of procedural memory. On the other hand, excessive shame responses are not healthy. Some types of narcissitic patients have excessive shame and they are inhibited in social situations. The model proposed by Kohut (Kohut 1984) it may be that narcissistic pathology develops when shaming experiences are too harsh. The sense of self feels diminished and the child's enthusiastic exploration is inhibited. These individuals defend against their shame, by disavowing or dissociating from it. As a result they are both over inhibited in some arenas social interaction and under inhibited in others. Although the person's longing for expansive expression of interest and enthusiasm remains split off, in the treatment it emerges in the transference.

Too much guilt is obviously not adaptive, and is essentially the basis for much of psychoanalytic treatment, most notably in the treatment of the classical neurosis. Guilt, involves an increase of SNS activity, as measured by the Galvanic Skin Response (an indicator of increased sweating and the basis of the 'lie detector' test). In some respects guilt is associated with fear and anxiety and is adaptive because it aids the individual in anticipating the negative consequences of their actions. Sociopaths, and certain brain damaged individuals, appear to have too little guilt. They lack the normal GSR response to situations of danger, and thus do not experience guilt (Damasio 1994). Sociopathic individuals are very poor psychoanalytic patients. They do not experience the necessary anxiety about the negative consequences of their behavior, even at an unconscious level. It appears that due to the lack of GSR response, maladaptive, even dangerous behavior remains ego syntonic and unlike the borderline patients whose ego-syntonic behavior is treatable, sociopaths lack the necessary bio-phyisology to shift over to an egodystonic perspective.

All analysts are well acquainted with patients who appear to be in a state of vigilance, on 'high alert' so to speak for danger, always perceiving other people as threatening, even the analyst who may never have said or done anything harmful. The neuroscience perspective helps explain such a transference. When a person is anxious or fearful, from whatever cause, a recent rape or a childhood trauma, or even an inborn temperamental trait, the brain's appraisal system is more likely to appraise 'in coming stimuli' as dangerous, leading potentially to the vicious cycle in which fear breeds even more fear. This is what typically occurs with PTSD. For example a physical assault or accident, generates 
fear. Once the person is afraid, their appraisal system is biased toward danger and they can develop a state of vigilance, in which they are more likely to interpret situations as dangerous. The most commonly known example, is the war veteran who shakes with fear to loud noises such as the back firing of a car. This is one of the best uses of neuroscience I have found. Trauma patients benefit from understanding this kind of cognitive bias, and can use it to re-interpret what they consider dangerous. Even in the transference, patients who have suffered trauma in childhood are on high alert to the dangers of being abandoned, or abused, and may perhaps startle, cringe or cower to otherwise neutral movements by the analyst - a shift in the chair, a tap of the foot, even merely sitting behind the patient.

But too little fear and anxiety is not good either. In the case mentioned earlier of the woman professional, she disavowed fear. Because of job responsibilities she was often required to travel to dangerous areas, where she would walk alone at night on isolated streets. Also as a result of her job, she was once stalked and threatend by a former employee but did not think of letting the authorities know. In the transference she said she was in a "protected bubble" with me. I would interpret to her that while such safety must be a good feeling, that without access to her own fear, she was not able to properly protect herself from danger. She replied that to deny fear, was a way of maintaining a tie to her mother even in the mother's absense. For her it was as if, mother would be there and save her. If she accepted fear, it would mean, she would have to experience being alone, without a mother who would come and rescue her.

\section{3. WHY WE SHOW OUR FEELINGS}

One reason we show our feelings, is simply to communicate to others what we are feeling. A bright smile can communicate to others that we are happy, tears that we are sad, in much the same as words communicate. But another reason, beyond communication per se, is that we are innately designed such that the expression of emotion by one person automatically and unconsciously regulates the behavior, emotion and body physiology of the person to whom it is expressed! This kind of mutual regulation occurs, because the non-verbal behaviors of emotion of one person, are assessed by the other's AMG and OFC appraisal centers, and in turn (through connections with the HYPO and BS) generate biochemical changes, ANS activity, and behaviors in the other. There 
are many examples of, how mutual regulation through the non-verbal behavior of emotion, is evident, through out life. The baby's cry of distress, is not simply to let mother know how the baby feels, it is biologically designed to activate the very nurturant care taking responses, such as hugging, holding and touching which will relieve the distress. This has been well studied in many animal models (Hofer 1996b; Kalin 1993; Kalin, Shelton et al. 1995) and is presumed to operate in a similar way in humans. When a child is too dampened down with shame for an extended period, this is designed to activate the parent to re-animate the child, with words, and perhaps touch, of encouragement or forgiveness (Schore 1994). Even between adults, when a person hangs their head in shame, this can serve to activate forgiveness in the other. As two strangers approach, if one stares directly into the eyes of the other, this is a sign of threat and the other backs off. If however, as they approach, one averts their eyes, this signals they are not a threat and that the other can approach. When a sad person cries, this activates sympathy in others. The clinical implication is that the patient's non-verbal behavior will activate these 'emotion-regulation' responses automatically and involuntarily in the analyst as well. The analysts own self reflection, as to how they feel inclined to respond, may be an indication of the emotion in the patient, even if the patient is not conscious of that emotion. The analyst's sensitivity to the patient's silence - how long to wait, when and how to intervene - probably derives from these kinds of non-verbal cues.

In certain respects, the neuroscience perspective supports the idea of projective identification. However, unlike the psychoanalytic concept, the biologic system of matching and empathy is innate, and automatic. It does not imply any defensive purposes, although, most likely this system can be used defensively. Whether the patient's non-verbal emotion behavior is a way to defend against conscious affects, or simply a reflection of those affects, it can activate "countertransferense" feeling states in the analyst that can be utilized to formulate an interpretation. One male patient, an attorney kept pressing me to have a sexual relationship, often trying to humiliate me for not being willing to have such an experience (Pally 2001). I had a pronounced feeling of disgust rather than feeling either flattered or sexually aroused myself. He would spout political and moral ideals and aggressively try to get me to agree with his ideas, in this case demeaning the psychoanalytic technique which prevents me from agreeing. I found his ideas more repugnant than agreeable. Aware that there was a lot of hostility in the transference, toward 
me as a woman and as an authority figure, and informed by my feeling of disgust, I finally interpreted, "It is as if you want to shove your ideas down my throat and make me swallow them even if I don't want to." Another male patient, a magazine editor, feels he deserves nothing and will aggravate people if he asks for help or support. He is always cheerful and engaging. He talks about his concerns as if he is telling an interesting story. Despite his charming flare for entertaining me, I continually feel inclined to reassure him, to offer words of encouragement and hope. It is as if, he activates 'comforting behavioral' responses in me, somehow by his non-verbal manner. While it could be argued that my need to comfort could reflect my own 'issues' so to speak, I think these kinds of inclinations in therapists are also part of the natural and normal way we as humans are designed to react to one another's distress, even if only expressed non-verbally and maybe even outside conscious awareness, and that analyst's can use these feelings in themselves as guides for transference interpretations.

One theory about why individuals with alexithymia often suffer dysregulated emotion is that they are not only poor at verbally expressing feelings, they are also poor at expressing the non-verbal behaviors of emotion. Therefore they are less likely to activate the kinds of responses in others which will serve to regulate them. For example even if they are quite sad or frightened, they may talk in a monotone with a bland face. For the therapist this can lead to boredom. Without the outwardly expressed non-verbal behavior of emotion, the therapist is not activated to either empathize or sympathize. The therapist neither 'gets' what the patient is feeling nor tries to comfort and soothe.

\section{4. WHY WE CONSCIOUSLY FEEL OUR FEELINGS}

The vast majority of brain processes are non-conscious, which includes the psychoanalytic concepts of unconscious (both the conflictual and conflict free aspects), as well as the pre-conscious. Only a very small percentage ever becomes conscious. Consciousness occurs 'after the fact'. First something is processed non-consciously and then, a few milliseconds later, can become available to consciousness. With respect to emotion, the brain first generates emotion non-consciously, and only afterward do we become aware of the emotional behavior and conscious awareness of a 'feeling'.

But what is the purpose of consciousness? This is as yet not well understood. One theory is that consciousness plays a role in the brain's 
oversight system (Posner and Rothbart 1998; Freeman 2000; Fuster 2003). In other words, consciousness may play a monitoring role, for determining whether a particular emotional response was adaptive. If an emotional response becomes conscious and the person recognizes the emotion as inappropriate the brain can 're-appraise' the situation and switch to a different emotion. Or the brain can inhibit the 'inappropriate' behavior which follows from that emotion and voluntarily initiate a more adaptive one. For example, I often find myself feeling tense when a patient has a long silence. I notice myself start to speak, subvocally. After I am aware of my mouth starting to move, or perhaps my body shift slightly, but before any words are actually uttered, I will realize I am responding more to my own tension, than to what the patient might need from me, and I inhibit my talking. In the case of one male patient, the magazine editor, whose transference involves a very high degree of wanting to meet my needs, he is hyper-alert to sensing these tiny movements of mine, and often, particularly in the beginning of the analysis, would stop his internal reverie and says to me, "Go ahead, say what you were going to say. It is alright." Now, well into the analysis, many times he is able to ignore my movements and to continue the silence or begin talking according to his own need.

Much of the time, emotion is activated entirely outside of awareness. This means we are not aware of an emotional 'feeling' and may not even be aware of any physiologic changes in our body, or even our motor actions, such as facial expression. Clinically this is significant, because without recognizing it, both patient and analyst can still effect each other's affect, physiology and behavior, albeit non-consciously.

The value of being conscious of feelings, particularly the uniquely human type of consciousness known as 'self-reflective consciousness', is that it appears to facilitate regulation of emotion. This is particularly relevant to psychoanalysis, since putting feelings into words, (a major part of the analytic treatment), enhances self-reflective consciousness. Humans, like all mammals, have the innate ability to be consciously aware in the moment. Only humans can be conscious of being aware and, can reflect on their experience (Edelman 1992; Edelman \& Tononi 2000; Damasio 1994). Such reflection helps an individual to recognize what aspect of the situation is stimulating their emotional response, and that there are a number of alternative ways of responding. Babies are born with basic 'animal' conscious awareness. As they develop linguistic ability, they can reflect more and more on their conscious awareness. Such reflection makes possible re-appraisal of a situation and the recog- 
nition of alternatives to one's initial response. I would argue that by enhancing self-refletive consciousness, verbalizing one's emotion, serves to enhance self-monitoring and to recognize whether one's initial response was appropriate and adaptive and to inhibit their behavioral response and try to take in more stimuli to see if it is possible to shift to another emotion with a different behavioral response. In analysis, by verbally expressing emotions, the patient is more able to reflect, rather than act out; more able to move outside of the regressed transference response and take a more mature stance; more able to utilize the observing ego, rather than just the experiencing ego. This helps explain why, even without any interpretive intervention, eventually most people feel better for having talked about their emotions. In some cases reflection on feelings helps patients to actually feel differently about a situation.

However, in many cases, the feelings are too engrained, too deeply rooted, and no amount of verbalizing and self-reflective consciousness can alter this. I think one of the most valuable lessons of neuroscience is that we cannot control what emotions we have. Emotion is activated involuntarily and out of our awareness, and that there is nothing maladaptive about any particular emotion per se. What can be maladaptive are the behaviors which result from an emotion. Conscious reflection on emotion, which is enhanced by putting words to emotions, even if it can't change the emotion, can help inhibit the maladaptive behavior and activate a more adaptive one, because, verbal expression and conscious reflection engages the PFC, the brain's center for abstract representation and selevtive choice of behavior (Fuster 2003) I make this kind of perspective explicit with patients. I tell them that, particularly with very painful experiences, such as occurs with the extremes of emotional and physical abuse, the treatment may not help them feel any less hurt, any less ashamed, any less angry (although I hope it does). It can however help them not act on those feelings. In the long run, by not acting, and by tolerating the feelings more, in fact they are morel likely to dissipate on their own (as emotions naturally do), and thus, in the end, feel less intense and painful.

\section{5. MEMORY AND DEFENSES}

The encoding of the long term memory for events, generally referred to as 'declarative' or 'explicit' memory, is strengthened by optimal levels of emotion (McGaugh 1995). When an event is appraised as 'emotionally significant', an increase of the neurotransmitter NE is re- 
leased in the brain, which activates the AMG to send a signal to the HIPPO to encode the memory. Conversely, when not enough emotion is generated, not enough NE will be released and long term memory for the event is only weakly encoded or not at all encoded. Once memory is encoded, it must be retrieved for us to be conscious of it. The stronger the encoding process, the more likely the memory will be able to be retrieved.

Too much and too little emotion actually impairs memory. At one end of the continuum, too much emotional arousal can interfere with the encoding of explicit memory. In this case the hormone 'cortisol' is involved. When cortisol gets very high, this can cause impairment or even cell death in the HIPPO. For example veterans exposed to war violence and children exposed to physical and sexual abuse show a smaller than normal size HIPPO (Bremner, Randal et al. 1995; Sapolsky 1996). What this implies clinically, is that many patients who appear to have 'repressed' their traumatic experiences, may in fact suffer from a biologic impairment of memory encoding.

At the other end, too little emotion can serve the purpose of impairing memory and recall of painful aspects of a situation (Edelman 1992; Fuster 1996; Edelman and Tononi 2000; Fuster 2003). Some patients utilize this for defensive purposes. One woman in treatment, a business analyst, claims that she had a happy childhood, but can't remember much of it. In the transference she seems to often feel criticized by me, but if I make a comment about it, she says "No I wasn't feeling that way". This woman uses the defense of disavowal of affect. Characteristically she might say "As a child I never could please my mother, but I didn't let it effect me". One way to understand her lack of childhood memories is that when a person ignores or minimizes the emotional impact of an event, this can impair their ability to store the event in long term memory in the first place, or to later recall it from long term memory. ${ }^{8}$

As important as it is to remember, it is equally important to forget! In fact, there are brain systems for forgetting. Under the usual circumstances, all memories gradually fade to some degree. Insignificant ones may fade away completely. For example, most likely, you will not remember tomorrow what you had for breakfast yesterday. Where as, significant ones may simply fade in intensity. This is why you no longer

\footnotetext{
${ }^{8}$ From a neuroscience perspective, what analysts refer to as repression, is the inability to retrieve memories that have been encoded.
} 
can remember the intensely stabbing pain you felt when you broke your leg several years ago.

Impaired 'forgetting' is one way of understanding the condition known as Post Traumatic Stress Disorder (PTSD) (Van der Kolk 1993). In PTSD the traumatic memory does not gradually fade. It remains vivid, as if occurring 'right now'. The biochemical response associated with PTSD helps to explain this disorder of 'forgetting'. CRF is released by the traumatic event, but, for reasons not yet fully understood, the cortisol response is blunted (i.e., not as high as would be expected for that level of stress). Since cortisol acts to shut off the initial NE response of emotional arousal, patients with PTSD are in a chronic state of high NE and SNS arousal, with anxiety, insomnia and hyperactive startle response, as well has highly vivid traumatic memories, that do not fade. In fact each time they 're-remember' the trauma in flashbacks, it becomes even more strongly encoded. The blunted cortisol found with trauma, may also be associated with impaired transmission of information from the right hemisphere, which processes emotion, to the left hemisphere, which processes language. These patients have emotion, but may appear emotionally detached, because they cannot verbalize what they feel. To treat the PTSD, it is necessary to help them experience their feelings and put them into words, which enables the traumatic memory to be integrated with the whole body of prior memory. In this way the memory fades into the past.

Defenses are mobilized to avoid painful affects. Research suggests a similar mechanism between managing physical pain and managing emotional pain (Sierra and Berrios 1998; Simeon, Guralnik et al. 2000). Pharmacologic studies and neuroimaging studies illustrate how the limbic system, may be employed to reduced physical pain and to activate psychological defenses. In the pharmacolgic treatment of pain, anesthetics and analgesics are used. Anesthetics, (e.g. Novocain), block all pain sensation. With analgesics, such as the narcotic morphine, the pain is still there, but it doesn't bother the person. It is known that analgesics reduce emotional pain as well as physical pain. A region of the limbic system, known as the Cingulate Cortex, is involved in pain awareness. Subjects are hypnotized and given either a suggestion for pain anesthesia or pain analgesia.. . If a person is given the hypnotic suggestion for anesthesia, neuroimaging reveals decreased activity in the cingulate and decreased in the somato-sensory cortex as well. If the suggestion is for analgesia, there is diminished activity only in the cingulated. Activitiy is normal in the somato-sensory cortex. The cingulate diminishes aware- 
ness of the pain, even though the sensory cortex still records the pain sensations! This suggests that the cingulate may also be employed in defenses against awareness of emotional pain such as disavowal and dissociation, . The PFC may also be involved in defenses. The PFC is the executive center of the brain and functions mostly to inhibit activity in lower centers. A case of a woman who suffered an emotional shock and developed a hysterical paralysis indicates the role of the PFC in the defense (Marshall, Halligan et al. 1997). After her arm became 'paralyzed', neuroimaging revealed low activity in the right motor cortex and high activity in the PFC. Presumably the PFC was inhibiting her motor cortex. In the case of organic paralysis due to stroke, neuroimaging typically shows low activity of motor cortex, but with normal activity in the PFC. If a subject is given a hypnotic suggestion for paralysis, the same neuroimaging profile as with 'hysterical' paralysis occurs. Putting this data together, what is suggested is that the effect of emotion on the PFC, as well as hypnotic suggestion may utilize similar neural circuits and may help explain certain aspects of repression. Emotion, or Hypnotic Suggestion, acting on the PFC, inhibits activity in the lower cortical sites where the memory is stored, and therefore sites receive insufficient activity for the memory to be retrieved (see footnote 7).

\section{6. RATIONAL THINKING IS NOT SO RATIONAL}

Neuroscientists now consider that phrases such as a "heart felt" decision or a "gut decision", may not just be metaphors (Damasio 1994). Everytime we have an experience, it activates emotion which activates the body. The OFC stores the memory of the emotion, including the body changes involved in that emotion, in association with the sensory, motor and contextual aspects of the experience. The body changes are referred to as the 'somatic marker' of the experience. The OFC uses the somatic markers as guides in the decision making process. When a person has to make a decision or a choice as to how to behave in a particular situation, the brain relies on how that decision affected the body in a similar past situation. In essence the brain relies on somatic markers to determine "How did I feel the last time I acted in such and such a way in this same type of situation". Patients with brain damage to the OFC and many individuals with sociopathy show impaired decision making, presumably because of impairments in their ability to assess somatic markers. It is assumed that sociopaths cannot learn from the negative consequences of past behavior because of the impairment in somatic marker 
registration. Clinically what this suggests is that for adaptive functioning, people need to have emotions about the events of their lives, to create somatic markers of experience, because this is how we learn from past experience.

\section{CONCLUSION}

Emotions are not just subjective feelings. Emotions are as much about the body as is digestion and respiration. What neuroscience indicates is that these bodily aspects of emotion are very relevant to the clinical issues we address in psychoanalysis. They help to explain many of the symptoms of psychological disorders, and why they are so deeply entrenched. They also help to explain how empathy, transference, countertransference and psychological defenses occur, and even address the patient's oft asked question "why do I have to feel my feelings?"

Although emotion is now center stage in neuroscience and in psychoanalysis, there is one caveat. Some emotions are so deeply engrained we cannot change them, even with the best analysis. These patients will continue to have the old painful feelings and will have the impulse or tendency to act on those feelings. I tell patients we have no control over how we feel, only over how we act. This need not be a negative outcome however, because patients in analysis, who are able to bring their emotions into conscious awareness, can learn to inhibit the maladaptive behavior which results from that emotion.

\section{ACKNOWLEDGMENTS}

I had help in the development of these ideas from the Westside Neuroscience Study Group whose members are Hans Miller, John Schumann, Leon Sones, John Sherin, Bob Agagenian, Don Faraveau and myself; from colleagues Dan Siegel, Allan Shore, David Olds and Beatrice Beebe; and The UCLA Brain Research Institute Journal Club headed by Joaquin Fuster and Arnold Scheibel. 


\section{REFERENCES}

Beebe, B. \& Lachmann, F.M. (1988). The contributions of mother-infant mutual influence to the origins of self and object representations. Psychoanalytic Psychology 5: 305-337.

Beebe, B. \& Lachmann, F.M. (1998). Co-constructing Inner and Relational Process. Psychoanalytic Psychology 15: 480-516.

Bremner, J.D., Randal, P., Scott, T.M., Bronen, R.A., Seibyl, J.P., Southwick, S.M., McCarthy, G., Charney, D. S. \& Innis, R. B. (1995). MRI-based measurement of hippocampal volume in patients with combat-related post-traumatic stress disorder. American Journal of Psychiatry 152: 973-981.

Damasio, A.R. (1994). Descartes Error. New York: Putnam.

Damasio, A.R. (1995). Toward a neurobiology of emotion and feeling: operational concepts and hypotheses. The Neuroscientists 1: 19-25.

Damasio, A.R. (1999). The Feeling of What Happens. New York: Harcourt.

Davidson, R.J., Jackson, D.C. \& Kalin, N.H. (2000). Emotion, plasticity, context and regulation: perspectives from affective neuroscience. Psychological Bulletin 126: 890-909.

Davidson, R.J., Putnam, K.M. \& Larson, C.L. (2000). Dysfunction in the neural circuitry of emotion regulation - A possible prelude to violence. Science 289: 591-594.

Davis, M. (1992). The role of the amygdala in fear and anxiety. Annual Reviews in Neuroscience. 15: 353-375.

DeGangi, G.A., DiPietro, J.A., Greenspan, S.I. \& Porges, S.W. (1991). Psychophysiological characteristics of the regulatory disordered infant. Infant Behavior and Development 14: 37-50.

Edelman, G. (1992). Bright Air, Brilliant Fire. New York: Basic Books.

Edelman, G.M. \& Tononi, G. (2000). A Universe of Consciousness: How Matter Becomes Imagination. New York: Basic Books.

Ekman, P. (1983). Autonomic nervous system activity distinguishes among emotions. Science 221: 1208-1210.

Ekman, P. (1990). Voluntary facial action generates emotion specific autonomic nervous system activity. Psychophysiology 27: 363-383.

Ekman, P. (1993). Facial expression and emotion. American Psychologist 48: 384-392.

Feldstein, S. \& Welkowitz, J., ed. (1978). A Chronography of Conversation. Nonverbal Behavior and Communication. Hillsdale: Lawrence Erlbaum Associates.

Freeman, W.J. (2000). How Brains Make Up Their Minds. New York: Columbia University Press.

Freud, S. (1895d) (Together with Breuer, J.). Studies on Hysteria. S. E. 2.

Freud, S. (1950a [1985]). Project for a scientific psychology. S.E. 1: 295-387.

Fuster, J.M., ed. (1996). Frontal Lobe and the Cognitive Foundation of Behavioral Action. Neurology of Decision Making. Berlin: Springer-Verlag.

Fuster, J.M. (2003). Prefrontal Cortex. Oxford, New York: Oxford University Press.

Grigsby, J. (1994). Procedural learning and the development and stability of character. Perceptual and Motor Skills 79: 355-370. 
Gunnar, M. (1992). Reactivity of the hypothalamic-pituitary-adrenocortical system to stressors in normal infants and children. Pediatrics 90: 491-497.

Henry, J.P. (1993). Psychological and physiological responses to stress: The right hemisphere and the hypothalamo-pituitary-adrenal axis, an inquiry into problems of human bonding. Physiological and Behavioral Science 28: 369-387.

Hofer, M. (1996a). On the nature and consequences of early loss. Psychosomatic Medicine 58: 570-581.

Hofer, M.A. (1996b). Multiple regulators of ultrasonic vocalization in the infant rat. Psychoneuroendocrinology 21: 203-217.

Insel, T. (1997). A neurobiological basis of social attachment. American Journal of Psychiatry. 154: 726-735.

Kalin, N. (1993). The neurobiology of fear. Scientific American 268: 94-101.

Kalin, N.H., Shelton, S.E., Lynn, D.E. (1995). Opiate systems in mother and infant primates coordinate intimate contact during reunion. Psychoneuroendocrinology 20: 735-42.

Kohut, H. (1984). How Does Analysis Cure? Chicago: The University of Chicago Press.

Krystal, H. (1974). The genetic development of affects and affect regression. The Annual of Psychoanalysis 2: 98-126.

LeDoux, J. (1994). Emotion, memory and the brain. Scientific American 270: 32-39.

LeDoux, J. (1995). Emotion: Clues from the brain. Annual Review of Psychology 46: 209-235.

LeDoux, J. (1996). The Emotional Brain. New York: Simon and Schuster.

Marshall, J.C., Halligan, P.W., Fink, G.R., Wade, D.T. \& Frackowiak, R.S.J. (1997). The functional anatomy of a hysterical paralysis. Cognition 64: B1-B8.

McGaugh, J.L. (1995). Emotional activation, neuromodulatory systems and memory. Memory Distortion: How Minds, Brains and Societies Reconstruct the Past, ed. D.L. Schacter. Cambridge: Harvard University Press: 255-273.

Meaney, M.J. (2001). Maternal care, gene expression, and the transmission of individual differences in stress reactivity across generations. Annual Reviews in Neuroscience 24: 1161-1192.

Nemeroff, C.B. (2002). Recent advances in the neurobiology of depression. Psychopharmacol Bull 200236 Supplement 2: 6-23.

Newport D.J., Stowe, Z.N. \& Nemeroff, C.B. (2002). Parental depression: animal models of an adverse life event. American Journal of Psychiatry 159: 1265-83.

Pally, R. (1996). Reciprocal Responsiveness in Psychoanalysis. Journal of Clinical Psychoanalysis. Proceedings of the New York Psychoanalytic Institute and Society 5: 7-23.

Pally, R. (2001). A Primary Role for Nonverbal Communication in Psychoanalysis. Psychoanalytic Inquiry 21: 71-93.

Panksepp, J. (1998). Affective Neuroscience. Oxford: Oxford University Press.

Posner, M.I. \& Rothbart, M.K. (1998). Attention, self-regulation and consciousness. Phil. Trans. R. Soc. Lond. B 353: 1915-1927.

Sapolsky, R.M. (1996). Why stress is bad for your brain. Science 273: 749-750.

Schore, A.N. (1994). Affect Regulation and the Origin of the Self. Hillsdale: Lawrence Erlbaum. 
Sierra, M. \& Berrios, G.E. (1998). Depersonalization: neurobiological perspectives. Biologic Psychiatry 44: 898-908.

Silk, K., ed. (1998). Biology of Personality Disorders. Review of Psychiatry. Washington: American Psychiatric Press.

Silk, K. (2000). Overview of biologic factors. Pediatric Clinics of North America 23: $61-75$.

Simeon, D., Guralnik, O., Hazlett, E.A., Spiegel-Cohen, J., Hollander, E. \& Buchsbaum, M.S. (2000). Feeling unreal: A PET study of depersonalization disorder. American Journal of Psychiatry 157(11): 1782-1788.

Stern, D. (1985). The Interpersonal World of the Infant. New York: Basic Books.

Suomi, S.J. (1999). Attachment in rhesus monkeys. In Handbook of Attachment: Theory, Research, and Clinical Application, ed. J. Cassidy \& P. R. Shaver. New York: Guilford Press, pp. 181-197.

Taylor, G.J. (1992). Psychoanalysis and psychosomatics: A new synthesis. Journal of the American Academy of Psychoanalysis and Dynamic Psychiatry 20: 251275.

Teicher, M.H. (2000). Wounds that won't heal: the neurobiology of child abuse. Cerebrum 2: 50-62.

Van der Kolk, B.A. (1993). Biological considerations about emotions, trauma, memory and the brain. In Human Feelings: Explorations in Affect Development and Meaning, ed. S.L. Ablon, D. Brown, E.J. Khantzian, \& J.E. Mack. Hillsdale: Analytic Press, pp. 221-240.

Winnicott, D. (1956). Primary maternal preoccupation. The Child and the Family. London: Tavistock 1957.

Yehuda, R. (2001). Biology of posttraumatic stress disorder. Journal of Clinical Psychiatry 62 Supplement 17: 41-46. 
